\title{
Asymptotic Proportion of Hard Instances of the Halting Problem
}

\author{
Antti Valmari*
}

\begin{abstract}
Although the halting problem is undecidable, imperfect testers that fail on some instances are possible. Such instances are called hard for the tester. One variant of imperfect testers replies "I don't know" on hard instances, another variant fails to halt, and yet another replies incorrectly "yes" or "no". Also the halting problem has three variants: does a given program halt on the empty input, does a given program halt when given itself as its input, or does a given program halt on a given input. The failure rate of a tester for some size is the proportion of hard instances among all instances of that size. This publication investigates the behaviour of the failure rate as the size grows without limit. Earlier results are surveyed and new results are proven. Some of them use $\mathrm{C}++$ on Linux as the computational model. It turns out that the behaviour is sensitive to the details of the programming language or computational model, but in many cases it is possible to prove that the proportion of hard instances does not vanish.
\end{abstract}

Keywords: halting problem, three-way tester, generic-case tester, approximating tester

\section{Introduction}

Turing proved in 1936 that undecidability exists by showing that the halting problem is undecidable [10]. Rice extended the set of known undecidable problems to cover all questions of the form "does the partial function computed by the given program have property $X$ ", where $X$ is any property that at least one computable partial function has and at least one does not have [7]. For instance, $X$ could be "returns 1 for all syntactically correct $\mathrm{C}++$ programs and 0 for all remaining inputs." In other words, it may be impossible to find out whether a given weirdlooking program is a correct $\mathrm{C}++$ syntax checker. These results are basic material in such textbooks as [3].

On the other hand, imperfect halting testers are possible. For any instance of the halting problem, a three-way tester eventually answers "yes", "no", or "I

\footnotetext{
*Tampere University of Technology, Department of Mathematics, PO Box 553, FI-33101 Tampere, FINLAND, E-mail: Antti.Valmari@tut.fi
} 
don't know". If it answers "yes" or "no", then it must be correct. We say that the "I don't know" instances are hard instances for the tester. Also other kinds of imperfect testers have been introduced, as will be discussed in Section 2.1.

Assume that $T_{1}$ is a tester. By Turing's proof, it has a hard instance $I_{1}$. If $I_{1}$ is a halting instance, then let $T_{2}$ be "if the input is $I_{1}$, then reply 'yes', otherwise run $T_{1}$ and return its reply". If $I_{1}$ is non-halting, then let $T_{2}$ be "if the input is $I_{1}$, then reply 'no', otherwise run $T_{1}$ and return its reply". By construction, $T_{2}$ is a tester with one fewer hard instances than $T_{1}$ has. By Turing's proof, also $T_{2}$ has a hard instance. Let us call it $I_{2}$. It is hard also for $T_{1}$. This reasoning can be repeated without limit, yielding an infinite sequence $T_{1}, T_{2}, \ldots$ of testers and $I_{1}, I_{2}, \ldots$ of instances such that $I_{i}$ is hard for $T_{1}, \ldots, T_{i}$ but not for $T_{i+1}, \ldots$ Therefore, every tester has an infinite number of hard instances, but no instance is hard for all testers.

A program that answers "I don't know" for every program and input is a threeway tester, although it is useless. A much more careful tester simulates the given program on the given input at most $9^{9^{n}}$ steps, where $n$ is the joint size of the program and its input. If the program stops by then, then the tester answers "yes". If the program repeats a configuration (that is, a complete description of the values of variables, the program counter, etc.) by then, then the tester answers "no". Otherwise it answers "I don't know". With this theoretically possible but in practice unrealistic tester, any hard halting instance has a finite but very long running time.

The proofs by Turing and Rice may leave the hope that only rare artificial contrived programs yield hard instances. One could dream of a three-way tester that answers very seldom "I don't know". This publication analyses this issue, by surveying and proving results that tell how the proportion of hard instances behaves when the size of the instances grows without limit.

Section 2 presents the variants of the halting problem and imperfect testers surveyed, together with some basic results and notation. Earlier research is discussed in Section 3. The section contains some proofs to bring results into the framework of this publication. Section 4 presents some new results in the case that a program has many copies of all big sizes, or information can be packed densely inside the program. It is not always assumed that the program has access to the information. A natural example of such information is dead code, such as if $(1==0)$ then $\{\ldots\}$. In Section 5, results are derived for $\mathrm{C}++$ programs with inputs from files. Section 6 briefly concludes this publication.

This publication is a significantly extended version of $[12,13]$. The papers $[12$, 13] are otherwise essentially the same, but three proofs were left out from [13] because of lack of space. In the present publication, Theorems 4 and 6 and Corollaries 2 and 4 are new results lacking from [12,13]. Furthermore, [12, 13] incorrectly claimed the opposite of Theorem 6. The present publication fixes this error and also a small error in Proposition 4. 


\section{Concepts and Notation}

\subsection{Variants of the Halting Problem}

The literature on hard instances of the halting problem considers at least three variants of the halting problem:

$\mathbf{E}$ does the given program halt on the empty input [2],

$\mathbf{S}$ does the given program halt when given itself as its input $[6,8]$, and

$\mathbf{G}$ does the given program halt on the given input $[1,4,9]$.

Each variant is undecidable. Variant $G$ has a different notion of instances from others: program-input pairs instead of just programs. A tester for G can be trivially converted to a tester for $\mathrm{E}$ or $\mathrm{S}$, but the proportion of hard program-input pairs among all program-input pairs of some size is not necessarily the same as the similar proportion with the input fixed to the empty one or to the program itself.

The literature also varies on what the tester does when it fails. Three-way testers, that is, the "I don't know" answer is used implicitly by [6], as it discusses the union of two decidable sets, one being a subset of the halting and the other of the non-halting instances. In generic-case decidability [8], instead of the "I don't know" answer, the tester itself fails to halt. Yet another idea is to always give a "yes" or "no" answer, but let the answer be incorrect for some instances $[4,9]$. Such a tester is called approximating. One-sided results, where the answer is either "yes" or "I don't know", were presented in [1, 2]. For a tester of any of the three variants, we say that an instance is easy if the tester correctly answers "yes" or "no" on it, otherwise the instance is hard.

These yield altogether nine different sets of testers, which we will denote with three-way $(\mathrm{X})$, generic $(\mathrm{X})$, and $\operatorname{approx}(\mathrm{X})$, where $\mathrm{X}$ is $\mathrm{E}, \mathrm{S}$, or G. Some simple facts facilitate carrying some results from one variant of testers to another.

Proposition 1. For any three-way tester there is a generic-case tester that has precisely the same easy "yes"-instances, easy "no"-instances, hard halting instances, and hard non-halting instances.

There also is an approximating tester that has precisely the same easy "yes"instances, at least the same easy "no"-instances, precisely the same hard halting instances, and no hard non-halting instances; and an approximating tester that has at least the same easy "yes"-instances, precisely the same easy "no"-instances, no hard halting instances, and precisely the same hard non-halting instances.

Proof. A three-way tester can be trivially converted to the promised tester by replacing the "I don't know" answer with an eternal loop, the reply "no", or the reply "yes".

Proposition 2. For any generic-case tester there is a generic-case tester that has at least the same "yes"-instances, precisely the same "no"-instances, no hard halting instances, and precisely the same hard non-halting instances. 
Proof. In parallel with the original tester, the instance is simulated. (In Turing machine terminology, parallel simulation is called "dovetailing".) If the original tester replies something, the simulation is aborted. If the simulation halts, the original tester is aborted and the reply "yes" is returned.

Proposition 3. For any $i \in \mathbb{N}$ and tester $T$, there is a tester $T_{i}$ that answers correctly "yes" or "no" for all instances of size at most $i$, and similarly to $T$ for bigger instances.

Proof. Because there are only finitely many instances of size at most $i$, there is a finite bit string that lists the correct answers for them. If $n \leq i, T_{i}$ picks the answer from it and otherwise calls $T$. (We do not necessarily know what bit string is the right one, but that does not rule out its existence.)

\subsection{Notation}

We use $\Sigma$ to denote the set of characters that are used for writing programs and their inputs. It is finite and has at least two elements. There are $|\Sigma|^{n}$ character strings of size $n$. If $\alpha$ and $\beta$ are in $\Sigma^{*}$, then $\alpha \sqsubseteq \beta$ denotes that $\alpha$ is a prefix of $\beta$, and $\alpha \sqsubset \beta$ denotes proper prefix. The size of $\alpha$ is denoted with $|\alpha|$.

A set $A$ of finite character strings is self-delimiting if and only if membership in $A$ is decidable and no member of $A$ is a proper prefix of a member of $A$. The shortlex ordering of any set of finite character strings is obtained by sorting the strings in the set primarily according to their sizes and strings of the same size in the lexicographic order.

Not necessarily all elements of $\Sigma^{*}$ are programs. The set of programs is denoted with $\Pi$, and the set of all (not necessarily proper) prefixes of programs with $\Gamma$. So $\Pi \subseteq \Gamma$. For tester variants $\mathrm{E}$ and $\mathrm{S}$, we use $p(n)$ to denote the number of programs of size $n$. Then $p(n)=\left|\Sigma^{n} \cap \Pi\right|$. For tester variant $\mathrm{G}, p(n)$ denotes the number of program-input pairs of joint size $n$. We will later discuss how the program and its input are paired into a single string. The numbers of halting and non-halting (a.k.a. diverging) instances of size $n$ are denoted with $h(n)$ and $d(n)$, respectively. We have $p(n)=h(n)+d(n)$.

If $T$ is a tester, then $\underline{h}_{T}(n), \bar{h}_{T}(n), \underline{d}_{T}(n)$, and $\bar{d}_{T}(n)$ denote the number of its easy halting, hard halting, easy non-halting, and hard non-halting instances of size $n$, respectively. Obviously $\underline{h}_{T}(n)+\bar{h}_{T}(n)=h(n)$ and $\underline{d}_{T}(n)+\bar{d}_{T}(n)=d(n)$. The smaller $\bar{h}_{T}(n)$ and $\bar{d}_{T}(n)$ are, the better the tester is. The failure rate of $T$ is $\left(\bar{h}_{T}(n)+\bar{d}_{T}(n)\right) / p(n)$.

When referring to all instances of size at most $n$, we use capital letters. So, for example, $P(n)=\sum_{i=0}^{n} p(i)$ and $\bar{D}_{T}(n)=\sum_{i=0}^{n} \bar{d}_{T}(i)$. 


\section{Related Work}

\subsection{Early Results by Lynch}

Nancy Lynch [6] used Gödel numberings for discussing programs. In essence, it means that each program has at least one index number (which is a natural number) from which the program can be constructed, and each natural number is the index of some program.

Although the index of an individual program may be smaller than the index of some shorter program, the overall trend is that indices grow as the size of the programs grows, because otherwise we would run out of small numbers. On the other hand, if the mapping between the programs and indices is $1-1$, then the growth cannot be faster than exponential. This is because $p(n) \leq|\Sigma|^{n}$. With real-life programming languages, the growth is exponential, but (as we will see in Section 5.2) the base of the exponent may be smaller than $|\Sigma|$.

To avoid confusion, we refrain from using the notation $\bar{H}_{T}$, etc., when discussing results in [6], because the results use indices instead of sizes of programs, and their relationship is not entirely straightforward. Fortunately, some results of [6] can be immediately applied to programming languages by using the shortlex Gödel numbering. The shortlex Gödel number of a program is its index in the shortlex ordering of all programs.

The first group of results of [6] reveals that a wide variety of situations may be obtained by spreading the indices of all programs sparsely enough and then filling the gaps in a suitable way. For instance, with one Gödel numbering, for each threeway tester, the proportion of hard instances among the first $i$ indices approaches 1 as $i$ grows. With another Gödel numbering, there is a three-way tester such that the proportion approaches 0 as $i$ grows. There even is a Gödel numbering such that as $i$ grows, the proportion oscillates in the following sense: for some three-way tester, it comes arbitrarily close to 0 infinitely often and for each three-way tester, it comes arbitrarily close to 1 infinitely often.

In its simplest form, spreading the indices is analogous to defining a new language SpaciousC ++ whose syntax is identical to that of $\mathrm{C}++$ but the semantics is different. If the first $\lfloor n / 2\rfloor$ characters of a SpaciousC ++ program of size $n$ are space characters, then the program is executed like a $\mathrm{C}++$ program, otherwise it halts immediately. This does not restrict the expressiveness of the language, because any $\mathrm{C}++$ program can be converted to a similarly behaving Spacious $\mathrm{C}++$ program by adding sufficiently many space characters to its front. However, it makes the proportion of easily recognizable trivially halting instances overwhelm. A program that replies "yes" if there are fewer than $\lfloor n / 2\rfloor$ space characters at the front and "I don't know" otherwise, is a three-way tester. Its proportion of hard instances vanishes as the size of the program grows.

As a consequence of this and Proposition 3, one may choose any failure rate above zero and there is a three-way tester for SpaciousC ++ programs with at most that failure rate. Of course, this result does not tell anything about how hard it is to test the halting of interesting programs. This is the first example in this 
publication of what we call an anomaly stealing the result. That is, a proof of a theorem goes through for a reason that has little to do with the phenomenon we are interested in.

Indeed, the first results of [6] depend on using unnatural Gödel numberings. They do not tell what happens with untampered programming languages. Even so, they rule out the possibility of a simple and powerful general theorem that applies to all models of computation. They also make it necessary to be careful with the assumptions that are made about the programming language.

To get sharper results, optimal Gödel numberings were discussed in [6]. They do not allow distributing programs arbitrarily. A Gödel numbering is optimal if and only if for any Gödel numbering, there is a computable function that maps it to the former such that the index never grows more than by a constant factor. ${ }^{1}$ The most interesting sharper results are opposite to what was obtained without the optimality assumption. To apply them to programming languages, we first define a programming language version of optimal Gödel numberings.

Definition 1. A programming language is end-of-file data segment, if and only if each program consists of two parts in the following way. The first part, called the actual program, is written in a self-delimiting language (so its end can be detected). The second part, called the data segment, is an arbitrary character string that extends to the end of the file. The language has a construct via which the actual program can read the contents of the data segment.

The data segment is thus a data literal in the program, packed with maximum density. It is not the same thing as the input to the program.

Corollary 1. For each end-of-file data segment language,

$$
\begin{aligned}
& \exists c>0: \exists T \in \operatorname{three-way}(\mathrm{S}): \forall n \in \mathbb{N}: \frac{\underline{H}_{T}(n)+\underline{D}_{T}(n)}{P(n)} \geq c \text { and } \\
& \exists c>0: \forall T \in \operatorname{three-way}(\mathrm{S}): \exists n_{T} \in \mathbb{N}: \forall n \geq n_{T}: \frac{\bar{H}_{T}(n)+\bar{D}_{T}(n)}{P(n)} \geq c .
\end{aligned}
$$

Proof. Let $\mathcal{L}$ be the end-of-file data segment language, and let $\mathcal{G}$ be any Gödel numbering. Consider the following program $P$ in $\mathcal{L}$. Let $a$ and $d$ be the sizes of its actual program and data segment. The actual program reads the data segment, interpreting its content as a number $i$ in the range from $\frac{|\Sigma|^{d}-1}{|\Sigma|-1}+1$ to $\frac{|\Sigma|^{d+1}-1}{|\Sigma|-1}$. Then it simulates the $i$ th program in $\mathcal{G}$. The shortlex index of $P$ is at most $i^{\prime}=$ $\sum_{j=0}^{a+d}|\Sigma|^{j} \leq|\Sigma|^{a+d+1}$. We have $\frac{|\Sigma|^{d}-1}{|\Sigma|-1}+1 \leq i$, yielding $|\Sigma|^{d}-1 \leq|\Sigma| i-i-|\Sigma|+1$, so $|\Sigma|^{d} \leq|\Sigma| i$, thus $i^{\prime} \leq|\Sigma|^{a+2} i$. The shortlex numbering of $\mathcal{L}$ is thus an optimal Gödel numbering. From this, Proposition 6 in [6] gives the claims.

\footnotetext{
${ }^{1}$ The definition in [6] seems to say that the function must be a bijection. We believe that this is a misprint, because each proof in [6] that uses optimal Gödel numberings obviously violates it.
} 
A remarkable feature of the latter result compared to many others in this publication is that $c$ is chosen before $T$. That is, there is a positive constant that only depends on the programming language (and not on the choice of the tester) such that all testers have at least that proportion of hard instances, for any big enough $n$. On the other hand, the proof depends on the programming language allowing to pack raw data very densely. Real-life programming languages do not satisfy this assumption. For instance, $\mathrm{C}++$ string literals " . . " cannot pack data densely enough, because the representation of " inside the literal (e.g., \" or \042) requires more than one character.

Because of Proposition 3, " $\exists n_{T} \in \mathbb{N}$ " cannot be moved to the front of " $\forall T \in$ three-way(S)" .

The result cannot be generalized to $\bar{h}_{T}, \bar{d}_{T}$, and $p$, because the following anomaly steals it. We can change the language by first adding 1 or 01 to the beginning of each program $\pi$ and then declaring that if the size of $1 \pi$ or $01 \pi$ is odd, then it halts immediately, otherwise it behaves like $\pi$. This trick does not invalidate optimality but introduces infinitely many sizes for which the proportion of hard instances is 0 .

\subsection{Results on Domain-Frequent Programming Languages}

In [4], the halting problem was analyzed in the context of programming languages that are frequent in the following sense:

Definition 2. A programming language is (a) frequent (b) domain-frequent, if and only if for every program $\pi$, there are $n_{\pi} \in \mathbb{N}$ and $c_{\pi}>0$ such that for every $n \geq n_{\pi}$, at least $c_{\pi} p(n)$ programs of size $n$ (a) compute the same partial function as $\pi$ (b) halt on precisely the same inputs as $\pi$.

Instead of "frequent", the word "dense" was used in [4], but we renamed the concept because we felt "dense" a bit misleading. The definition says that programs that compute the same partial function are common. However, the more common they are, the less room there is for programs that compute other partial functions, implying that the smallest programs for each distinct partial function must be distributed more sparsely. "Dense" was used for domain-frequent in [9].

Any frequent programming language is obviously domain-frequent but not necessarily vice versa. On the other hand, even if a theorem in this field mentions frequency as an assumption, the odds are that its proof goes through with domainfrequency. Whether a real-life programming language such as $\mathrm{C}++$ is domainfrequent, is surprisingly difficult to find out. We will discuss this question briefly in Section 4.1.

As an example of a frequent programming language, BF was mentioned in [4]. Its full name starts with "brain" and then contains a word that is widely considered inappropriate language, so we follow the convention of [4] and call it BF. Information on it can be found on Wikipedia under its real name. It is an exceptionally simple programming language suitable for recreational and illustrational but not for reallife programming purposes. In essence, BF programs describe Turing machines with 
a read-only input tape, write-only output tape, and one work tape. The alphabet of each tape is the set of 8-bit bytes. However, BF programs only use eight characters.

As a side issue, a non-trivial proof was given in [4] that only a vanishing proportion of character strings over the eight characters are BF programs. That is, $\lim _{n \rightarrow \infty} p(n) / 8^{n}$ exists and is 0 . It trivially follows that if all character strings over the 8 characters are considered as instances and failure to compile is considered as non-halting, then the proportion of hard instances vanishes as $n$ grows.

The only possible compile-time error in BF is that the square brackets [ and ] do not match. Most, if not all, real-life programming languages have parentheses or brackets that must match. So it seems likely that compile-time errors dominate also in the case of most, if not all, real-life programming languages. Unfortunately, this is difficult to check rigorously, because the syntax and other compile-time rules of real-life programming languages are complicated. Using another, simpler line of argument, we will prove the result for both $\mathrm{C}++$ and BF in Section 5.1.

In any event, if the proportion of hard instances among all character strings vanishes because the proportion of programs vanishes, that is yet another example of an anomaly stealing the result. It is uninteresting in itself, but it rules out the possibility of interesting results about the proportion of hard instances of size $n$ among all character strings of size $n$. Therefore, from now on, excluding Section 5.1, we focus on the proportion of hard instances among all programs or program-input pairs.

In the case of program-input pairs, the results may be sensitive to how the program and its input are combined into a single string that is used as the input of the tester. To avoid anomalous results, it was assumed in $[4,9]$ that this "pairing function" has a certain property called "pair-fair". The commonly used function $x+(x+y)(x+y+1) / 2$ is pair-fair. To use this pairing function, strings are mapped to numbers and back via their indices in the shortlex ordering of all finite character strings.

A proof was sketched in [9] that, assuming domain-frequency and pair-fairness,

$$
\forall T \in \operatorname{approx}(\mathrm{G}): \exists c_{T}>0: \exists n_{T} \in \mathbb{N}: \forall n \geq n_{T}: \frac{\bar{h}_{T}(n)+\bar{d}_{T}(n)}{p(n)} \geq c_{T}
$$

That is, the proportion of wrong answers does not vanish. However, this leaves open the possibility that for any failure rate $c>0$, there is a tester that fares better than that for all big enough $n$. This possibility was ruled out in [4], assuming frequency and pair-fairness. (It is probably not important that frequency instead of domainfrequency was assumed.) That is, there is a positive constant such that for any tester, the proportion of wrong answers exceeds the constant for infinitely many sizes of instances:

$$
\exists c>0: \forall T \in \operatorname{approx}(\mathrm{G}): \forall n_{0} \in \mathbb{N}: \exists n \geq n_{0}: \frac{\bar{h}_{T}(n)+\bar{d}_{T}(n)}{p(n)} \geq c .
$$

The third main result in [4], adapted and generalized to the present setting, is the following. We present its proof to obtain the generalization and to add a detail 
that the proof in [4] lacks, that is, how $T_{i, j}$ is made to halt for "wrong sizes". Generic-case testers are not mentioned, because Proposition 2 gave a related result for them.

Theorem 1. For each programming model and variant E, S, G of the halting problem,

$$
\begin{aligned}
& \forall c>0: \exists T_{c} \in \operatorname{approx}(\mathrm{X}) \quad: \forall n_{0} \in \mathbb{N}: \exists n \geq n_{0}: \frac{\bar{h}_{T_{c}}(n)}{p(n)} \leq c \wedge \frac{\bar{d}_{T_{c}}(n)}{p(n)}=0 \text { and } \\
& \forall c>0: \exists T_{c} \in \operatorname{three-way}(\mathrm{X}): \forall n_{0} \in \mathbb{N}: \exists n \geq n_{0}: \frac{\bar{h}_{T_{c}}(n)}{p(n)} \leq c .
\end{aligned}
$$

Proof. Let $C=\lceil 1 / c\rceil$. Consider the family $T_{i, j}$ of the programs of the following kind, where $i \in \mathbb{N}, j \in \mathbb{N}$, and $0 \leq i \leq C$. If $n<j, T_{i, j}$ answers "no" in the case of approximating and "I don't know" in the case of three-way testers. If $n \geq j, T_{i, j}$ simulates all instances of size $n$ until $\lceil i p(n) / C\rceil$ of them have halted. If the simulation stage terminates, then if the given instance is among those that halted, $T_{i, j}$ answers "yes", otherwise $T_{i, j}$ answers "no" or "I don't know". Thus an approximating $T_{i, j}$ has $\bar{d}_{T_{i, j}}(n)=0$.

We prove next that some $T_{i, j}$ is the required tester. Let $i_{n}=\lfloor C h(n) / p(n)\rfloor$. Then $i_{n} p(n) / C \leq h(n)<\left(i_{n}+1\right) p(n) / C$. When $n \geq j$, the simulation stage of $T_{i_{n}, j}$ terminates and the proportion of hard halting instances of $T_{i_{n}, j}$ is less than $1 / C \leq c$. Some $0 \leq i \leq C$ is the $i_{n}$ for infinitely many values of $n$. Furthermore, there is a smallest such $i$. We denote it with $i^{\prime}$. There also is a $j$ such that when $n \geq j$, then $i_{n} \geq i^{\prime}$. With these choices, $T_{i^{\prime}, j}$ always halts.

For a small enough $c$ and the approximating tester $T_{c}$ in Theorem 1, (1) implies that the failure rate of $T_{c}$ oscillates, that is, does not approach any limit as $n \rightarrow \infty$. This observation is directly obtainable from Lemma 23 in [4].

\subsection{Results on Turing Machines}

For Turing machines with one-way infinite tape and randomly chosen transition function, the probability of falling off the left end of the tape before halting or repeating a state approaches 1 as the number of states grows [2]. The tester simulates the machine until it falls off the left end, halts, or repeats a state. If falling off the left end is considered as halting, then the proportion of hard instances vanishes as the size of the machine grows. This can be thought of as yet another example of an anomaly stealing the result.

Formally, $\exists T \in$ three-way $(\mathrm{X}): \lim _{n \rightarrow \infty}\left(\bar{h}_{T}(n)+\bar{d}_{T}(n)\right) / p(n)=0$, that is,

$$
\exists T \in \operatorname{three-way}(\mathrm{X}): \forall c>0: \exists n_{c} \in \mathbb{N}: \forall n \geq n_{c}: \frac{\bar{h}_{T}(n)+\bar{d}_{T}(n)}{p(n)} \leq c .
$$

Here X may be E, S, or G. Although E was considered in [2], the proof also applies to $\mathrm{S}$ and G. Comparing the result to Theorem 2 in Section 4.1 reveals that 
the representation of programs as transition functions of Turing machines is not domain-frequent.

On the other hand, independently of the tape model, the proportion does not vanish exponentially fast [8]. Like in [2], the proportion is computed on the transition functions, and not on some textual representations of the programs. The proof relies on the fact that any Turing machine has many obviously similarly behaving copies of bigger and bigger sizes. They are obtained by adding new states and transitions while keeping the original states and transitions intact. So the new states and transitions are unreachable. They are analogous to dead code. These copies are not common enough to satisfy Definition 2, but they are common enough to rule out exponentially fast vanishing. Generic-case decidability was used in [8], but the result applies also to three-way testers by Proposition 1.

The results in [1] are based on using weighted running times. For every positive integer $k$, the proportion of halting programs that do not halt within time $k+c$ is less than $2^{-k}$, simply because the proportion of times greater than $k+c$ is less than $2^{-k}$. The publication presents such a weighting that $c$ is a computable constant.

Assume that programs are represented as self-delimiting bit strings on the input tape of a universal Turing machine. The smallest three-way tester of variant E that answers "yes" or "no" up to size $n$ and "I don't know" for bigger programs, is of size $n \pm O(1)[11]$.

\section{Programming Languages with Assumptions}

\subsection{Domain-Frequent Languages}

The assumption that the programming language is domain-frequent (Definition 2) makes it possible to use a small variation of the standard proof of the non-existence of halting testers, to prove that each halting tester of variant $\mathrm{S}$ has a non-vanishing set of hard instances. For three-way and generic-case testers, one can also say something about whether the hard instances are halting or not. Despite its simplicity, as far as we know, the following result has not been presented in the literature. However, see the comment on [9] in Section 3.2.

Theorem 2. If the programming language is domain-frequent, then

$$
\begin{aligned}
& \forall T \in \text { three-way(S) }: \exists c_{T}>0: \exists n_{T} \in \mathbb{N}: \forall n \geq n_{T}: \frac{\bar{h}_{T}(n)}{p(n)} \geq c_{T} \wedge \frac{\bar{d}_{T}(n)}{p(n)} \geq c_{T}, \\
& \forall T \in \operatorname{generic}(\mathrm{S}): \exists c_{T}>0: \exists n_{T} \in \mathbb{N}: \forall n \geq n_{T}: \frac{\bar{d}_{T}(n)}{p(n)} \geq c_{T}, \text { and } \\
& \forall T \in \operatorname{approx}(\mathrm{S}): \exists c_{T}>0: \exists n_{T} \in \mathbb{N}: \forall n \geq n_{T}: \frac{\bar{h}_{T}(n)+\bar{d}_{T}(n)}{p(n)} \geq c_{T} .
\end{aligned}
$$

Proof. Let the execution of $X$ with an input $y$ be denoted with $X(y)$. For any $T$, consider the program $P_{T}$ that first tries its input $x$ with $T$. If $T(x)$ replies 
"yes", then $P_{T}(x)$ enters an eternal loop. If $T(x)$ replies "no", then $P_{T}(x)$ halts immediately. The case that $T(x)$ replies "I don't know" is discussed below. If $T(x)$ fails to halt, then $P_{T}(x)$ cannot continue and thus also fails to halt.

By the definition of domain-frequent, there are $c_{T}>0$ and $n_{T} \in \mathbb{N}$ such that when $n \geq n_{T}$, at least $c_{T} p(n)$ programs halt on precisely the same inputs as $P_{T}$. Let $P^{\prime}$ be any such program. Consider $P_{T}\left(P^{\prime}\right)$. If $T\left(P^{\prime}\right)$ answers "yes", then $P_{T}\left(P^{\prime}\right)$ fails to halt. Then also $P^{\prime}\left(P^{\prime}\right)$ fails to halt. Thus "yes" cannot be the correct answer for $T\left(P^{\prime}\right)$. A similar reasoning reveals that also "no" cannot be the correct answer for $T\left(P^{\prime}\right)$. So $P^{\prime}$ is a hard instance for $T$.

Nothing more is needed to prove the claim for approximating testers. In the case of generic-case testers, the hard instances make $T$ and thus $P_{T}$ fail to halt, so they are non-halting instances.

In the case of three-way testers, all hard instances can be made halting instances by making $P_{T}$ halt when $T$ replies "I don't know". This proves the claim $\bar{h}_{T}(n) / p(n) \geq c_{T}$. The claim $\bar{d}_{T}(n) / p(n) \geq c_{T}$ is proven by making $P_{T}$ enter an eternal loop when $T$ replies "I don't know". These two proofs may yield different $c_{T}$ values, but the smaller one of them is suitable for both. Similarly, the bigger of their $n_{T}$ values is suitable for both.

The second claim of Theorem 2 lacks a $\bar{h}_{T}(n)$ part. Indeed, Proposition 2 says that with generic-case testers, $\bar{h}_{T}(n)$ can be made 0 . With approximating testers, $\bar{h}_{T}(n)$ can be made 0 at the cost of $\bar{d}_{T}(n)$ becoming $d(n)$, by always replying "yes". Similarly, $\bar{d}_{T}(n)$ can be made 0 by always replying "no".

The next theorem applies to testers of variant $\mathrm{E}$ and presents some results similar to Theorem 2. To our knowledge, it is the first theorem of its kind that applies to the halting problem on the empty input. It assumes not only that many enough equivalent copies exist but also that they can be constructed. On the other hand, its equivalence only pays attention to the empty input.

Definition 3. A programming language is computably empty-frequent if and only if there is a decidable equivalence relation " $\approx$ " between programs such that

- for each program $\pi$, there are $c_{\pi}>0$ and $n_{\pi} \in \mathbb{N}$ such that for every $n \geq n_{\pi}$, at least $c_{\pi} p(n)$ programs of size $n$ are equivalent to $\pi$, and

- for each programs $\pi$ and $\pi^{\prime}$, if $\pi \approx \pi^{\prime}$, then either both or none of $\pi$ and $\pi^{\prime}$ halt on the empty input.

If $\pi \approx \pi^{\prime}$, we say that $\pi^{\prime}$ is a cousin of $\pi$.

It can be easily seen from [4] that BF is computably empty-frequent.

Theorem 3. If the programming language is computably empty-frequent, then

$$
\forall T \in \text { three-way(E) }: \exists c_{T}>0: \exists n_{T} \in \mathbb{N}: \forall n \geq n_{T}: \frac{\bar{d}_{T}(n)}{p(n)} \geq c_{T} .
$$

The result also holds for generic-case testers but not for approximating testers. 
Proof. Given any three-way tester $T$, consider a program $P_{T}$ that behaves as follows. First it constructs its own code and stores it in a string variable. Hard-wiring the code of a program inside the program is somewhat tricky, but it is well known that it can be done. With Gödel numberings, the same can be obtained with Kleene's second recursion theorem.

Then $P_{T}$ starts constructing its cousins of all sizes and tests each of them with $T$. By the assumption, there are $c_{T}>0$ and $n_{T} \in \mathbb{N}$ such that for every $n \geq n_{T}$, $P_{T}$ has at least $c_{T} p(n)$ cousins of size $n$. If $T$ ever replies "yes", then $P_{T}$ enters an eternal loop and thus does not continue testing its cousins. If $T$ ever replies "no", then $P_{T}$ halts immediately. If $T$ replies "I don't know", then $P_{T}$ tries the next cousin.

If $T$ ever replies "yes", then $P_{T}$ fails to halt on the empty input. By definition, also the tested cousin fails to halt on the empty input. So the answer "yes" would be incorrect. Similarly, if $T$ ever replies "no", that would be incorrect. So $T$ must reply "I don't know" for all cousins of $P_{T}$. They are thus hard instances for $T$. Because there are infinitely many of them, $P_{T}$ does not halt, so they are non-halting.

To prove the result for generic-case testers, it suffices to run the tests of the cousins in parallel, that is, go around a loop where each test that has been started is executed one step and the next test is started. If any test ever replies "yes" or "no", $P_{T}$ aborts all tests that it has started and then does the opposite of the reply.

A program that always replies "no" is an approximating tester with $\bar{d}_{T}(n)=0$ for every $n \in \mathbb{N}$.

The results in this section and Section 3.2 motivate the question: are real-life programming languages domain-frequent? For instance, is $\mathrm{C}++$ domain-frequent? Unfortunately, we have not been able to answer it. We try now to illustrate why it is difficult.

Given any $\mathrm{C}++$ program, it is easy to construct many longer programs that behave in precisely the same way, by adding space characters, line feeds (denoted with $\triangleleft)$, comments, or dead code such as if $(0 !=0)\{\ldots\}$. It is, however, hard to verify that many enough programs are obtained in this way. For instance, it might seem that many enough programs can be constructed with string literals. We now provide evidence that suggests (but does not prove) that it fails.

Any program of size $n$ can be converted to $(|\Sigma|-3)^{k}$ identically behaving programs of size $n+k+12$ by adding $\{\operatorname{char} * \mathbf{s}=" \sigma "$; $\}$ to the beginning of some function, where $\sigma \in(\Sigma \backslash\{", \backslash, \downarrow\})^{k}$. (The purpose of $\{$ and $\}$ is to hide the variable s, so that it does not collide with any other variable with the same name.) More programs are obtained by including escape codes such as \" to $\sigma$.

However, it seems that this is a vanishing instead of at least a positive constant proportion when $k \rightarrow \infty$. In the absence of escape codes, it certainly is a vanishing proportion. This is because one can add $\{$ char $* \mathrm{~s}=" \sigma ", * \mathrm{t}=" \rho "$; $\}$ instead, where $|\sigma|+|\rho|=k-6$. Without escape codes, this yields $(k-5)(|\Sigma|-3)^{k-6}$ programs. When $k \rightarrow \infty,(|\Sigma|-3)^{k} /\left((k-5)(|\Sigma|-3)^{k-6}\right)=(|\Sigma|-3)^{6} /(k-5) \rightarrow 0$.

That is, although string literals can represent information rather densely, they do not constitute the densest possible way of packing information into a $\mathrm{C}++$ 
program (assuming the absence of escape codes). A pair of string literals yields an asymptotically strictly denser packing. Similarly, a triple of string literals is denser still, and so on. Counting the programs in the presence of escape codes is too difficult, but it seems likely that the phenomenon remains the same.

So string literals do not yield many enough programs. It seems difficult to first find a construct that does yield many enough programs, and then prove that it works.

\subsection{End-of-file Data Segment Languages}

In this section we prove a theorem that resembles Theorem 3, but relies on different assumptions and has a different proof.

We say that a three-way tester is n-perfect if and only if it does not answer "I don't know" when the size of the instance is at most $n$. The following lemma is adapted from [11].

Lemma 1. Each programming language has a constant e such that the size of each $n$-perfect three-way tester of variant $E$ or $S$ is at least $n-e$.

Proof. Let $T_{n}$ be any $n$-perfect three-way tester of variant E or S. Consider a program $P$ that constructs character strings $x$ in shortlex order and tests them with $T_{n}$ until $T_{n}(x)$ replies "I don't know". If $T_{n}(x)$ replies "yes", $P$ simulates $x$ before trying the next character string. When simulating $x, P$ gives it the empty input in the case of variant $\mathrm{E}$ and $x$ as the input in the case of $\mathrm{S}$. The reply "I don't know" eventually comes, because otherwise $T_{n}$ would be a true halting tester. As a consequence, $P$ eventually halts. Before halting, $P$ simulates at least all halting programs of size at most $n$.

The time consumption of any simulated execution is at least the same as the time consumption of the corresponding genuine execution. So the execution of $P$ cannot contain properly a simulated execution of $P$. $P$ does not read any input, so it does not matter whether it is given itself or the empty string as its input. Therefore, the size of $P$ is bigger than $n$. Because the only part of $P$ that depends on $n$ is $T_{n}$, there is a constant $e$ such that the size of $T_{n}$ is at least $n-e$.

In any everyday programming language, space characters can be added freely between tokens. Motivated by this, we define that a blank character is a character that, for any program, can be added to at least one place in the program without affecting the meaning of the program.

Theorem 4. Let $X$ be $E$ or $S$. If the programming language is end-of-file data segment and has a blank character, then

$$
\forall T \in \operatorname{three-way}(\mathrm{X}): \exists c_{T}>0: \exists n_{T} \in \mathbb{N}: \forall n \geq n_{T}: \frac{\bar{h}_{T}(n)}{p(n)} \geq c_{T} \wedge \frac{\bar{d}_{T}(n)}{p(n)} \geq c_{T} .
$$

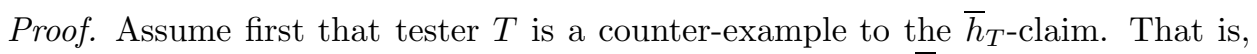
for every $c>0, T$ has infinitely many values of $n$ such that $\bar{h}_{T}(n) / p(n)<c$. 
If $T$ uses its data segment, let the use be replaced by the use of ordinary constants, liberating the data segment for the use described in the sequel. Let $T_{k, m}$ be the following program. Here $k$ is a constant inside $T_{k, m}$ represented by $\Theta(\log k)$ characters, and $m$ is the content of the data segment of $T_{k, m}$ interpreted as a natural number $m$ in base $|\Sigma|$. Let $a$ and $d$ be the sizes of the actual program and data segment of $T_{k, m}$. We have $a=\Theta(\log k)$. Let $x$ be the input of $T_{k, m}$.

The program $T_{k, m}$ first computes $n:=k+d$. If $|x|<n$, then $T_{k, m}$ adds blank characters to $x$, to make its size $n$. Next, if $|x|>n$, then $T_{k, m}$ replies "I don't know" and halts. Otherwise $T_{k, m}$ gives $x$ (which is now of size precisely $n$ ) to $T$. If $T(x)$ replies "yes" or "no", then $T_{k, m}$ gives the reply as its own reply and halts. Otherwise $T_{k, m}$ constructs each character string $y$ of size $n$ and tests it with $T$. $T_{k, m}$ simulates in parallel those $y$ for which $T(y)$ returns "I don't know" until $m$ of them have halted (with $y$ or the empty string as the input, as appropriate). Then it aborts those that have not halted. If $x$ is among those that halted, then $T_{k, m}$ replies "yes", otherwise $T_{k, m}$ replies "no".

For each $k \in \mathbb{N}$, there are infinitely many values of $n$ such that $\bar{h}_{T}(n) / p(n)<$ $|\Sigma|^{-k}$. For any such $n$ we have $\bar{h}_{T}(n)<p(n)|\Sigma|^{-k} \leq|\Sigma|^{n}|\Sigma|^{-k}$. So $n-k$ characters suffice for representing $\bar{h}_{T}(n)$. Therefore, there is $T_{k, m}$ such that $d=n-k$ and $m=\bar{h}_{T}(n)$. It is an $n$-perfect three-way tester of size $a+d=d+\Theta(\log k)=$ $n-k+\Theta(\log k)$. A big enough $k$ yields a contradiction with Lemma 1 .

The proof of the $\bar{d}_{T}$-claim is otherwise similar, but $T_{k, m}$ counts the number $v$ of those $y$ for which $T(y)$ returns "I don't know", and simulates the $y$ until $v-m$ of them have halted. The $\bar{h}_{T}$-claim and $\bar{d}_{T}$-claim are combined into a single claim by choosing the smaller $c_{T}$ and bigger $n_{T}$ provided by their proofs.

\subsection{End-of-file Dead Segment Languages}

In this section we show that if dead information can be added extensively enough, a tester of variant $\mathrm{E}$ with an arbitrarily small positive failure rate exists, but the opposite holds for variant $\mathrm{S}$. The reason for the result on variant $\mathrm{E}$ is that as the size of the programs grows, a bigger and bigger proportion of programs consists of copies of smaller programs. This phenomenon is so strong that to obtain the desired failure rate, it suffices to know the empty-input behaviour of all programs up to a sufficient size.

An end-of-file dead segment language is defined otherwise like end-of-file data segment language (Definition 1), but the actual program cannot read the data segment. This is the situation with any self-delimiting real-life programming language, whose compiler stops reading its input when it has read a complete program. Any end-of-file dead segment language is frequent and computationally domain-frequent.

Theorem 5. For each end-of-file dead segment language,

$$
\forall c>0: \exists T_{c} \in \operatorname{three-way}(\mathrm{E}): \forall n \in \mathbb{N}: \frac{\bar{h}_{T_{c}}(n)+\bar{d}_{T_{c}}(n)}{p(n)} \leq c .
$$

The result also holds with approximating and generic-case testers. 
Proof. Let $r(n)$ denote the number of programs whose dead segment is not empty. We have $r(n) \leq p(n) \leq|\Sigma|^{n}$, so $r(n)|\Sigma|^{-n} \leq 1$. For each $n \in \mathbb{N}, r(n+1)=$ $|\Sigma| p(n) \geq|\Sigma| r(n)$. So $r(n)|\Sigma|^{-n}$ grows as $n$ grows. These imply that there is $\ell$ such that $r(n)|\Sigma|^{-n} \rightarrow \ell$ from below when $n \rightarrow \infty$.

Because there are programs, $\ell>0$. For every $c>0$ we have $\ell c>0$, so there is $n_{c} \in \mathbb{N}$ such that $r\left(n_{c}\right)|\Sigma|^{-n_{c}} \geq \ell-\ell c$. On the other hand, $p(n)=r(n+1) /|\Sigma| \leq$ $\ell|\Sigma|^{n}$. These imply $p\left(n_{c}-1\right)|\Sigma|^{n-n_{c}+1} / p(n)=r\left(n_{c}\right)|\Sigma|^{n-n_{c}} / p(n) \geq 1-c$. Here $p\left(n_{c}-1\right)|\Sigma|^{n-n_{c}+1}$ is the number of those programs of size $n$ whose actual program is of size less than $n_{c}$.

The behaviour of a program on the empty input only depends on its actual program. Let $n_{a}$ be the size of the actual program. Consider a three-way tester that looks the answer from a look-up table if $n_{a}<n_{c}$ and replies "I don't know" if $n_{a} \geq n_{c}$ (cf. Proposition 3). It has $\left(\underline{h}_{T}(n)+\underline{d}_{T}(n)\right) / p(n) \geq 1-c$, implying the claim.

Proposition 1 generalizes the result to approximating and generic-case testers.

The above proof exploited the fact that the correct answer for a long program is the same as the correct answer for a similarly behaving short program. This does not work for testers of variant $\mathrm{S}$, because the short and long program no longer get the same input, since each one gets itself as its input. Although the program does not have direct access to its dead segment, it gets it via the input. This changes the situation to the opposite of the previous theorem.

Theorem 6. For each end-of-file dead segment language,

$$
\begin{aligned}
& \exists c>0: \forall T \in \text { three-way(S) }: \forall n_{0} \in \mathbb{N}: \exists n \geq n_{0}: \frac{\bar{h}_{T}(n)}{p(n)} \geq c \wedge \frac{\bar{d}_{T}(n)}{p(n)} \geq c, \\
& \exists c>0: \forall T \in \operatorname{generic}(\mathrm{S}) \quad: \forall n_{0} \in \mathbb{N}: \exists n \geq n_{0}: \frac{\bar{d}_{T}(n)}{p(n)} \geq c, \text { and } \\
& \exists c>0: \forall T \in \operatorname{approx}(\mathrm{S}) \quad: \forall n_{0} \in \mathbb{N}: \exists n \geq n_{0}: \frac{\bar{h}_{T}(n)+\bar{d}_{T}(n)}{p(n)} \geq c .
\end{aligned}
$$

Proof. We prove first the claims on three-way and generic-case testers.

Let us recall the overall idea of the proof of Theorem 2. In that proof, for any tester $T$, a program $P_{T}$ was constructed that gives its input $x$ to $T$. If $T(x)$ replies "yes", then $P_{T}(x)$ enters an eternal loop. If $T(x)$ replies "no", then $P_{T}(x)$ halts immediately. To prove that a three-way tester has many hard (a) halting (b) non-halting instances, in the case of the "I don't know" reply, $P_{T}(x)$ was made to (a) halt immediately (b) enter an eternal loop. All programs that halt on the same inputs as $P_{T}$ were shown to be hard instances for $T$. For each $n$ that is greater than a threshold that may depend on $T$, the existence of at least $c_{T} p(n)$ such programs was proven, where $c_{T}$ may depend on $T$ but not on $n$.

We now apply the same idea, but, to get a result where the same constant $c$ applies to all testers $T$, we no longer construct a separate program $P_{T}$ for each $T$. 
Instead, we construct a single program $P$, which obtains $T$ from the size of the input of $P$. (A similar idea appears in [4].) To discuss this, for any $i>0$, let $P_{i}$ be the program whose shortlex index is $i$. Let $\delta(i)=i-s(i)+1$, where $s(i)$ is the biggest square number that is at most $i$. The essence of $\delta(i)$ is that as $i$ gets the values $1,2,3, \ldots, \delta(i)$ gets each value $1,2,3, \ldots$ infinitely many times.

One more idea needs to be explained before discussing the details of $P$. Let $\Sigma$ be partitioned to $\Sigma_{1}$ and $\Sigma_{2}$ of sizes $\left\lfloor\frac{|\Sigma|}{2}\right\rfloor$ and $\left\lceil\frac{|\Sigma|}{2}\right\rceil$. Let $n_{a}$ be the size of the actual program of $P$. For each $n>n_{a}$, by modifying the dead segment, $|\Sigma|^{n-n_{a}}$ programs are obtained that have the same actual program as $P$. For $i \in\{1,2\}$, let $\Pi_{i}$ be the set of those of them whose dead segment ends with a character in $\Sigma_{i}$. We have $\frac{1}{3}|\Sigma|^{n-n_{a}} \leq\left|\Pi_{1}\right| \leq\left|\Pi_{2}\right|$. Because $0<p(n) \leq|\Sigma|^{n}$, by choosing $c=\frac{1}{3}|\Sigma|^{-n_{a}}$ we get $\frac{1}{3}|\Sigma|^{n-n_{a}} / p(n) \geq c$.

The program $P$ first checks that its input $x$ is a program with a non-empty dead segment. If it is not, then $P$ halts immediately. Otherwise, $P$ constructs $P_{\delta(|x|)}$ by going through all character strings in the shortlex order until $\delta(|x|)$ programs have been found. Then $P$ constructs every program $y$ that has the same size, has the same actual program, and belongs to the same $\Pi_{i}$ as $x$. Then $P$ executes the $P_{\delta(|x|)}(y)$ in parallel until any of the following happens.

If any $P_{\delta(|x|)}(y)$ replies "yes", then $P$ enters an eternal loop. If any $P_{\delta(|x|)}(y)$ replies "no", then $P$ aborts the remaining $P_{\delta(|x|)}(y)$ and halts. If every $P_{\delta(|x|)}(y)$ replies "I don't know", then $P$ halts if $x \in \Pi_{1}$, and enters an eternal loop if $x \in \Pi_{2}$. If none of the above ever happens, then $P$ fails to halt.

Recall that $n_{a}$ is the size of the actual program of $P$. For any tester $T$, there are infinitely many $n$ such that $n>n_{a}$ and $P_{\delta(n)}$ is $T$. For any such $n$, there are $|\Sigma|^{n-n_{a}}$ programs $P^{\prime}$ of size $n$ that have the same actual program as $P$. Let $P^{\prime \prime}$ be any of them. The execution of $P\left(P^{\prime \prime}\right)$ starts $P_{\delta(n)}\left(P^{\prime}\right)$ for at least $\frac{1}{3}|\Sigma|^{n-n_{a}}$ distinct $P^{\prime}$. If $P_{\delta(n)}\left(P^{\prime}\right)$ replies "yes", then $T$ claims that $P^{\prime}\left(P^{\prime}\right)$ halts. Then also $P\left(P^{\prime}\right)$ halts, because $P$ halts on the same inputs as $P^{\prime}$, since they have the same actual program. Furthermore, $P\left(P^{\prime \prime}\right)$ halts, because $P$ only looks at the size, actual program, and $\Pi_{i}$-class of its input, and $P^{\prime \prime}$ and $P^{\prime}$ agree on them. But the halting of $P\left(P^{\prime \prime}\right)$ is in contradiction with the behaviour of $P$ described above. Therefore, no $P_{\delta(n)}\left(P^{\prime}\right)$ can reply "yes". For a similar reason, none of them replies "no" either.

In conclusion, all at least $\frac{1}{3}|\Sigma|^{n-n_{a}}$ distinct $P^{\prime}$ are hard instances for $T$. If $T$ is a three-way tester, it replies "I don't know" for all of them. Depending on whether $P^{\prime \prime} \in \Pi_{1}$ or $P^{\prime \prime} \in \Pi_{2}$, they are hard halting or hard non-halting instances. If $T$ is a generic-case tester, it halts on none of these hard instances. Therefore, also $P\left(P^{\prime \prime}\right)$ and $P^{\prime \prime}\left(P^{\prime \prime}\right)$ fail to halt. So they all are hard non-halting instances.

In the case of approximating testers, $P$ is modified such that it lets all $P_{\delta(|x|)}(y)$ run into completion and counts the "yes"- and "no"-replies that they give. If the majority of the replies are "no", then $P$ halts, otherwise $P$ enters an eternal loop. For the same reasons as above, $P\left(P^{\prime \prime}\right)$ halts if and only if $P\left(P^{\prime}\right)$ halts if and only if $P^{\prime}\left(P^{\prime}\right)$ halts. So at least half of the replies are wrong.

Finally, we prove a corollary of the above theorem that deals with the halting problem itself, not with imperfect testers. Imperfect testers are used in the proof 
of the corollary, but not in the statement of the corollary.

Lemma 2. Let $X$ be any of $E, S$, and $G$, and let $f$ be any total computable function from natural numbers to integers. If

$$
\exists c>0: \forall T \in \operatorname{three-way}(\mathrm{X}): \forall n_{0} \in \mathbb{N}: \exists n \geq n_{0}: \frac{\bar{h}_{T}(n)}{p(n)} \geq c,
$$

then $\lim _{n \rightarrow \infty} \frac{h(n)-f(n)}{p(n)}$ does not exist.

Proof. Assume that $\lim _{n \rightarrow \infty}(h(n)-f(n)) / p(n)=x$ and $c>0$. Let $i=\left\lceil-\log _{2} c\right\rceil$. There is an $x_{i}$ of the form $m+\sum_{j=1}^{i+1} b_{j} 2^{-j}$ such that $m$ is an integer, $b_{j} \in\{0,1\}$ when $1 \leq j \leq i+1$, and $x_{i}<x \leq x_{i}+2^{-i-1}$. There also is $n_{0}$ such that when $n \geq n_{0}$, then $x_{i} \leq(h(n)-f(n)) / p(n)<x_{i}+2^{-i}$.

A tester $T$ that disobeys the formula is obtained as follows. If $n<n_{0}, T$ replies "I don't know". If $n \geq n_{0}, T$ simulates all instances of size $n$ until $\left\lceil x_{i} p(n)\right\rceil+f(n)$ have halted. If the given instance is among those that halted, then $T$ replies "yes" and otherwise "I don't know". We have $\bar{h}_{T}(n) / p(n)<2^{-i} \leq c$.

Corollary 2. Consider variant $S$ of the halting problem and any end-of-file dead segment language. Then $\lim _{n \rightarrow \infty} h(n) / p(n)$ does not exist.

The proof of Lemma 2 can be modified to approximating testers with $\left(\bar{h}_{T}(n)+\right.$ $\left.\bar{d}_{T}(n)\right) / p(n) \geq c$. By $(1)$, the limit fails to exist also in the framework of [4].

\section{$5 \mathrm{C}++$ without Comments and with Input}

\subsection{The Effect of Compile-Time Errors}

We first show that among all character strings of size $n$, those that are not $\mathrm{C}++$ programs - that is, those that yield a compile-time error - dominate overwhelmingly, as $n$ grows. In other words, a random character string is not a $\mathrm{C}++$ program except with vanishing probability. The result may seem obvious until one realizes that a $\mathrm{C}++$ program may contain comments and string literals which may contain almost anything. We prove the result in a form that also applies to BF.

$\mathrm{C}++$ is not self-delimiting. After a complete $\mathrm{C}++$ program, there may be, for instance, definitions of new functions that are not used by the program. This is because a $\mathrm{C}++$ program can be compiled in several units, and the compiler does not check whether the extra functions are needed by another compilation unit. Even so, if $\pi$ is a $\mathrm{C}++$ program, then $\pi 0$ is definitely not a $\mathrm{C}++$ program and not even a prefix of a $\mathrm{C}++$ program. Similarly, if $\pi$ is a BF program, then $\pi$ ] is not a prefix of a BF program.

Proposition 4. If for every $\pi \in \Pi$ there is $c \in \Sigma$ such that $\pi c \notin \Gamma$, then

$$
\lim _{n \rightarrow \infty} \frac{p(n)}{|\Sigma|^{n}}=0
$$


Proof. Let $q(n)=\left|\Sigma^{n} \cap \Gamma\right|$. Obviously $0 \leq p(n) \leq q(n) \leq|\Sigma|^{n}$.

Assume first that for every $\varepsilon>0$, there is $n_{\varepsilon} \in \mathbb{N}$ such that $p(n) / q(n)<\varepsilon$ for every $n \geq n_{\varepsilon}$. Because $0 \leq p(n) /|\Sigma|^{n} \leq p(n) / q(n)$, we get $p(n) /|\Sigma|^{n} \rightarrow 0$ as $n \rightarrow \infty$.

In the opposite case there is $\varepsilon>0$ such that $p(n) / q(n) \geq \varepsilon$ for infinitely many values of $n$. Let they be $n_{1}<n_{2}<\ldots$. Because $\pi c$ is not a prefix of any program, $q\left(n_{i}+1\right) \leq|\Sigma| q\left(n_{i}\right)-p\left(n_{i}\right) \leq(|\Sigma|-\varepsilon) q\left(n_{i}\right)$. For the remaining values of $n$, obviously $q(n+1) \leq|\Sigma| q(n)$. These imply that when $n>n_{i}$, we have $0 \leq p(n) /|\Sigma|^{n} \leq$ $q(n) /|\Sigma|^{n} \leq q\left(n_{i}\right) /|\Sigma|^{n_{i}} \leq(1-\varepsilon /|\Sigma|)^{i} \rightarrow 0$ when $i \rightarrow \infty$, which happens when $n \rightarrow \infty$.

Consider a tester $T$ that replies "no" if the compilation fails and "I don't know" otherwise. If compile-time error is considered as non-halting, then Proposition 4 implies that $\underline{h}_{T}(n) \rightarrow 0, \bar{h}_{T}(n) \rightarrow 0, \underline{d}_{T}(n) \rightarrow 1$, and $\bar{d}_{T}(n) \rightarrow 0$ when $n \rightarrow \infty$. As we pointed out in Section 3.2, this is yet another instance of an anomaly stealing the result.

\subsection{The $\mathrm{C}++$ Language Model}

The model of computation we study in this section is program-input pairs, where the programs are written in $\mathrm{C}++$, and the inputs obey the rules stated by the Linux operating system. Furthermore, $\Sigma$ is the set of all 8-bit bytes. To make firm claims about details, it is necessary to fix some language and operating system. The validity of the details below has been checked with $\mathrm{C}++$ and Linux. Most likely many other programming languages and operating systems could have been used instead.

There are two deviations from the real everyday programming situation. First, of course, it must be assumed that unbounded memory is available. Otherwise everything would be decidable. (However, at any instant of time, only a finite number of bits are in use.) Second, it is assumed that the programs do not contain comments. This assumption needs a discussion.

Comments are information that is inside the program but ignored by the compiler. They have no effect to the behaviour of the compiled program. We show next that most long $\mathrm{C}++$ programs consist of a shorter $\mathrm{C}++$ program and one or more comments.

Lemma 3. There are at most $(|\Sigma|-1)^{n}$ comment-less $C++$ programs of size $n$.

Proof. Everywhere inside a $\mathrm{C}++$ program excluding comments, it is either the case that $@$ or the case that the new line character $\lrcorner$ cannot occur next. That is, for every character string $\alpha$, either $\alpha @$ or $\alpha \triangleleft$ is not a prefix of any comment-less $\mathrm{C}++$ program.

(Perhaps surprisingly, there indeed are places that are outside comments and where any byte except $\lrcorner$ can occur.) 
Lemma 4. If $n \geq 16$, then there are at least $\left((|\Sigma|-1)^{4}+1\right)^{(n-19) / 4} C++$ programs of size $n$.

Proof. Let $A=\Sigma \backslash\{*\}$, and let $m=\lfloor n / 4-4\rfloor=\lceil(n-19) / 4\rceil$. Consider the character strings of the form

$$
\text { int } \operatorname{main}()\{/ * \alpha \beta * /\}
$$

where $\alpha$ consists of $(n \bmod 4)$ space characters and $\beta$ is any string of the form $\beta_{1} \beta_{2} \cdots \beta_{m}$, where $\beta_{i} \in A^{4} \cup\{* / / *\}$ for $1 \leq i \leq m$. Each such string is a syntactically correct $\mathrm{C}++$ program of size $n$. Their number is $\left((|\Sigma|-1)^{4}+1\right)^{m} \geq$ $\left((|\Sigma|-1)^{4}+1\right)^{(n-19) / 4}$.

Corollary 3. The proportion of comment-less $C_{++}$programs among all $C_{++}$ programs of size $n$ approaches 0 , when $n \rightarrow \infty$.

Proof. Let $s=|\Sigma|-1$. By Lemmas 3 and 4 , the proportion is at most $s^{n} /\left(s^{4}+1\right)^{(n-19) / 4}=s^{19}\left(s^{4} /\left(s^{4}+1\right)\right)^{(n-19) / 4} \rightarrow 0$, when $n \rightarrow \infty$.

As a consequence, although comments are irrelevant for the behaviour of programs, they have a significant effect on the distribution of long $\mathrm{C}++$ programs. To avoid the risk that they cause yet another anomaly stealing the result, we restrict ourselves to $\mathrm{C}++$ programs without comments. This assumption does not restrict the expressive power of the programming language, but reduces the number of superficially different instances of the same program.

The input may be any finite string of bytes. This is how it is in Linux. Although not all such inputs can be given directly via the keyboard, they can be given by directing the so-called standard input to come from a file. There is a separate test construct in $\mathrm{C}++$ for detecting the end of the input, so the end of the input need not be distinguished by the contents of the input. There are $256^{n}$ different inputs of size $n$.

The sizes of a program and input are the number of bytes in the program and the number of bytes in the input file. This is what Linux reports. The size of an instance is their sum. Analogously to Section 4.1, the size of a program is additional information to the concatenation of the program and the input. This is ignored by our notion of size. However, the notion is precisely what programmers mean with the word. Furthermore, the convention is similar to the convention in ordinary (as opposed to self-delimiting) Kolmogorov complexity theory [5].

Lemma 5. With the $C++$ programming model in Section 5.2, $p(n)<|\Sigma|^{n+1}$.

Proof. By Lemma 3, the number of different program-input pairs of size $n$ is at most

$$
\sum_{i=0}^{n}(|\Sigma|-1)^{i}|\Sigma|^{n-i}=|\Sigma|^{n} \sum_{i=0}^{n}\left(\frac{|\Sigma|-1}{|\Sigma|}\right)^{i}<|\Sigma|^{n} \sum_{i=0}^{\infty}\left(\frac{|\Sigma|-1}{|\Sigma|}\right)^{i}=|\Sigma|^{n+1} .
$$




\subsection{Proportions of Hard Instances}

The next theorem says that with halting testers of variant $\mathrm{G}$ and comment-less $\mathrm{C}++$, the proportions of hard halting and hard non-halting instances do not vanish.

Theorem 7. With the $C++$ programming model in Section 5.2,

$$
\forall T \in \operatorname{three-way}(\mathrm{G}): \exists c_{T}>0: \exists n_{T} \in \mathbb{N}: \forall n \geq n_{T}: \frac{\bar{h}_{T}(n)}{p(n)} \geq c_{T} \wedge \frac{\bar{d}_{T}(n)}{p(n)} \geq c_{T}
$$

Proof. We prove first the $\bar{h}_{T}(n) / p(n) \geq c_{T}$ part and then the $\bar{d}_{T}(n) / p(n) \geq c_{T}$ part. The results are combined by picking the bigger $n_{T}$ and the smaller $c_{T}$.

There is a program $P_{T}$ that behaves as follows. First, it gets its own size $n_{p}$ from a constant in its program code. The constant uses some characters and thus affects the size of $P_{T}$. However, the size of a natural number constant $m$ is $\Theta(\log m)$ and grows in steps of zero or one as $m$ grows. Therefore, by starting with $m=1$ and incrementing it by steps of one, it eventually catches the size of the program, although also the latter may grow.

Then $P_{T}$ reads the input, counting the number of the characters that it gets with $n_{i}$ and interpreting the string of characters as a natural number $x$ in base $|\Sigma|$. We have $0 \leq x<|\Sigma|^{n_{i}}$, and any natural number in this range is possible. Let $n=n_{p}+n_{i}$.

Next $P_{T}$ constructs every program-input pair of size $n$ and tests it with $T$. In this way $P_{T}$ gets the number $\underline{h}_{T}(n)$ of easy halting pairs of size $n$.

Then $P_{T}$ constructs again every pair of size $n$. This time it simulates each of them in parallel until $\underline{h}_{T}(n)+x$ of them have halted. Then it aborts the rest and halts. It halts if and only if $\underline{h}_{T}(n)+x \leq h(n)$. (It may be helpful to think of $x$ as a guess of the number of hard halting pairs.)

Among the pairs of size $n$ is $P_{T}$ itself with the string that represents $x$ as the input. We denote it with $\left(P_{T}, x\right)$. The time consumption of any simulated execution is at least the same as the time consumption of the corresponding genuine execution. So the execution of $\left(P_{T}, x\right)$ cannot contain properly a simulated execution of $\left(P_{T}, x\right)$. Therefore, either $\left(P_{T}, x\right)$ does not halt, or the simulated execution of $\left(P_{T}, x\right)$ is still continuing when $\left(P_{T}, x\right)$ halts. In the former case, $h(n)<\underline{h}_{T}(n)+x$. In the latter case $\left(P_{T}, x\right)$ is a halting pair but not counted in $\underline{h}_{T}(n)+x$, so $h(n)>\underline{h}_{T}(n)+x$. In both cases, $x \neq h(n)-\underline{h}_{T}(n)$.

As a consequence, no natural number less than $|\Sigma|^{n_{i}}$ is $\bar{h}_{T}(n)$. So $\bar{h}_{T}(n) \geq$ $|\Sigma|^{n_{i}}=|\Sigma|^{n-n_{p}}$. By Lemma 5, $p(n)<|\Sigma|^{n+1}$. So for any $n \geq n_{p}$, we have $\bar{h}_{T}(n) / p(n)>|\Sigma|^{-n_{p}-1}$.

The proof of the $\bar{d}_{T}(n) / p(n) \geq c_{T}$ part is otherwise similar, except that $P_{T}$ continues simulation until $p(n)-\underline{d}_{T}(n)-x$ pairs have halted. (Now $x$ is a guess of $\bar{d}_{T}(n)$, yielding a guess of $h(n)$ by subtraction.) The program $P_{T}$ gets $p(n)$ by counting the pairs of size $n$ whose program part is compilable. It turns out that $p(n)-\underline{d}_{T}(n)-x \neq h(n)$, so $x$ cannot be $\bar{d}_{T}(n)$, yielding $\bar{d}_{T}(n) \geq|\Sigma|^{n_{i}}$.

Next we adapt the second main result in [4] to our present setting, with a 
somewhat simplified proof and obtaining the result also for three-way and genericcase testers.

Theorem 8. With the $C++$ programming model in Section 5.2,

$$
\begin{aligned}
& \exists c>0: \forall T \in \operatorname{three-way}(\mathrm{G}): \forall n_{0} \in \mathbb{N}: \exists n \geq n_{0}: \frac{\bar{h}_{T}(n)}{p(n)} \geq c \wedge \frac{\bar{d}_{T}(n)}{p(n)} \geq c, \\
& \exists c>0: \forall T \in \operatorname{generic}(\mathrm{G}) \quad: \forall n_{0} \in \mathbb{N}: \exists n \geq n_{0}: \frac{\bar{d}_{T}(n)}{p(n)} \geq c, \text { and } \\
& \exists c>0: \forall T \in \operatorname{approx}(\mathrm{G}) \quad: \forall n_{0} \in \mathbb{N}: \exists n \geq n_{0}: \frac{\bar{h}_{T}(n)+\bar{d}_{T}(n)}{p(n)} \geq c .
\end{aligned}
$$

Proof. The proof follows the same strategy as the proof of Theorem 6, but differs in some technical details.

To prove the claim for three-way testers, for any character string $\alpha$, let $\operatorname{lb}(\alpha)=0$ if $\alpha$ is the empty string, and otherwise $\operatorname{lb}(\alpha)$ is the value of the least significant bit of the last character of $\alpha$. For any character strings $\alpha$ and $\beta$, let $\alpha \simeq \beta$ if and only if $|\alpha|=|\beta|$ and $\operatorname{lb}(\alpha)=\mathrm{lb}(\beta)$. For any size $n$ greater than 0 , " $\simeq$ " has two equivalence classes, each containing $|\Sigma|^{n} / 2$ character strings. For any $i>0$, let $P_{i}$ be the program whose shortlex index is $i$.

There is a program $P$ that behaves as follows. We denote its execution on input $\alpha$ with $P(\alpha)$. Please observe that if $\alpha \simeq \beta$, then $P(\beta)$ behaves in the same way as $P(\alpha)$.

First $P(\alpha)$ finds the program $P_{\delta(|\alpha|)}$, where $\delta(i)=i-s(i)+1$, where $s(i)$ is the biggest square number that is at most $i$.

Then $P(\alpha)$ goes through, in the shortlex order, all $\left\lceil|\Sigma|^{|\alpha|} / 2\right\rceil$ character strings $\beta$ such that $\alpha \simeq \beta$, until any of the termination conditions mentioned below occurs or $P(\alpha)$ has gone through all of them. For each $\beta$, it runs $P_{\delta(|\alpha|)}$ on $\beta$. We denote this with $P_{\delta(|\alpha|)}(\beta)$. If $P_{\delta(|\alpha|)}(\beta)$ fails to halt, then $P(\alpha)$ never returns from it and thus fails to halt. If $P_{\delta(|\alpha|)}(\beta)$ halts replying "yes", then $P(\alpha)$ enters an eternal loop, thus failing to halt. If $P_{\delta(|\alpha|)}(\beta)$ halts replying "no", then $P(\alpha)$ halts immediately. If $P_{\delta(|\alpha|)}(\beta)$ halts replying "I don't know", then $P(\alpha)$ tries the next $\beta$. It is not important what $P(\alpha)$ does if $P_{\delta(|\alpha|)}(\beta)$ halts replying something else.

If $P_{\delta(|\alpha|)}(\beta)$ halted replying "I don't know" for every $\beta$ such that $\alpha \simeq \beta$, then $P(\alpha)$ checks whether $\operatorname{lb}(\alpha)=0$. If yes, then $P(\alpha)$ enters an eternal loop, otherwise $P(\alpha)$ halts.

Now let $T(Q, \gamma)$ be any three-way tester that tests whether program $Q$ halts on the input $\gamma$. How the two components $Q$ and $\gamma$ of the input of $T$ are encoded into one input string is not important. There is a program that has $P$ hard-coded into a string constant, inputs $\beta$, calls $T(P, \beta)$, and gives its reply as its own reply. Let $i$ be the shortlex index of this program, so the program is $P_{i}$.

There are infinitely many positive integers $j$ such that $\delta(j)=i$. Let $j$ be such, and let $\alpha$ be any character string of size $j$. So $P_{\delta(|\alpha|)}$ is $P_{i}$. If, during the execution of $P(\alpha), P_{i}(\beta)$ ever replies "yes" or "no", then the same happens during 
the execution of $P(\beta)$, because $P(\beta)$ behaves in the same way as $P(\alpha)$ (the fact that $P_{i}(\beta)$ was called implies $\left.\alpha \simeq \beta\right)$. But that would be incorrect by the construction of $P$. Therefore, $T(P, \beta)$ replies "I don't know" for every $\beta$ of size $j$.

As a consequence, $T$ has at least $|\Sigma|^{j}$ hard instances of size $|P|+j$. If $j>0$, then half of them are halting and the other half non-halting, thanks to the $\operatorname{lb}(\alpha)=0$ test near the end of $P$. By Lemma $5, p(n)<|\Sigma|^{n+1}$. So if $n=|P|+j>|P|$, then

$$
\frac{\bar{h}_{T}(n)}{p(n)} \geq \frac{|\Sigma|^{n-|P|}}{2|\Sigma|^{n+1}}=\frac{1}{2|\Sigma|^{|P|+1}} \quad \text { and } \quad \frac{\bar{d}_{T}(n)}{p(n)} \geq \frac{1}{2|\Sigma|^{|P|+1}} .
$$

The program $P$ does not depend on $n$, so letting $c=1 /\left(2|\Sigma|^{|P|+1}\right)$ we have the claim.

The proof for generic-case testers is otherwise similar, but the $\beta$ are tried in parallel and $T(P, \beta)$ fails to halt for every $\beta$ of size $j$. All hard instances are non-halting. The $P$ for approximating testers lets each $P_{\delta(|\alpha|)}(\beta)$ continue until completion, counts the numbers of the "yes"- and "no"-replies they yield, and then does the opposite of the majority of the replies.

Application of Lemma 2 to this result yields the following.

Corollary 4. With the $C++$ programming model in Section 5.2, $\lim _{n \rightarrow \infty} h(n) / p(n)$ does not exist.

\section{Conclusions}

This study did not cover all combinations of a programming model, variant of the halting problem, and variant of the tester. So there is a lot of room for future work.

The results highlight what was already known since [6]: the programming model has a significant role. With some programming models, a phenomenon of secondary interest dominates the distribution of programs, making hard instances rare. Such phenomena include compile-time errors and falling off the left end of the tape of a Turing machine.

Many results were derived using the assumption that information can be packed very densely in the program or the input file. Sometimes it was not even necessary to assume that the program could use the information. It sufficed that the assumption allowed to make many enough similarly behaving longer copies of an original program. Intuition suggests that if the program can access the information, testing halting is harder than in the opposite case. A comparison of Theorem 5 to Theorem 6 supports this intuition.

Corollaries 2 and 4 and the comment after Corollary 2 tell that the proportion of all (not just hard) halting instances has no limit with end-of-file dead segment languages and variant $\mathrm{S}$ of the halting problem, with the $\mathrm{C}++$ model and variant $\mathrm{G}$, and in the framework of [4]. It must thus oscillate irregularly as the size of the program grows - irregularly because of Lemma 2. This is not a property of various notions of imperfect halting testers, but a property of the halting problem itself. 


\section{Acknowledgements}

I thank professor Keijo Ruohonen for helpful discussions, and the anonymous reviewers of SPLST ' 13 and Acta Cybernetica for their helpful comments. The latter pointed out that Proposition 4 had been formulated incorrectly.

\section{References}

[1] Calude, C. S. and Stay, M. A. Most programs stop quickly or never halt. Advances in Applied Mathematics, 40:295-308, 2008.

[2] Hamkins, J. D. and Miasnikov, A. The halting problem is decidable on a set of asymptotic probability one. Notre Dame Journal of Formal Logic, 47(4):515$524,2006$.

[3] Hopcroft, J. E. and Ullman, J. D. Introduction to Automata Theory, Languages, and Computation. Addison-Wesley, 1979.

[4] Köhler, S., Schindelhauer, C., and Ziegler, M. On approximating real-world halting problems. In Liśkiewicz, M. and Reischuk, R., editor, Proc. 15th Fundamentals of Computation Theory, Lecture Notes in Computer Science 3623, pages 454-466, 2005. Springer.

[5] Li, M. and Vitányi, P. An Introduction to Kolmogorov Complexity and Its Applications. Springer-Verlag, 2008.

[6] Lynch, N. Approximations to the halting problem. Journal of Computer and System Sciences, 9:143-150, 1974.

[7] Rice, H. G. Classes of recursively enumerable sets and their decision problems. Transactions of the American Mathematical Society 74:358-366, 1953.

[8] Rybalov, A. On the strongly generic undecidability of the halting problem. Theoretical Computer Science, 377:268-270, 2007.

[9] Schindelhauer, C. and Jakoby, A. The non-recursive power of erroneous computation. In Pandu Rangan, C., Raman, V., and Ramanujam, R., editors, Proc. 19th Foundations of Software Technology and Theoretical Computer Science, Lecture Notes in Computer Science 1738, pages 394-406, 1999. Springer.

[10] Turing, A. M. On computable numbers, with an application to the Entscheidungsproblem. Proceedings of the London Mathematical Society ser. 2, 42:230$265,1937$.

[11] Valmari, A. Sizes of up-to- $n$ halting testers. In Halava, V., Karhumäki, J., and Matiyasevich, Y., editors, Proceedings of the Second Russian Finnish Symposium on Discrete Mathematics, TUCS Lecture Notes 17, pages 176-183, Turku, Finland, 2012. 
[12] Valmari, A. The asymptotic behaviour of the proportion of hard instances of the halting problem (extended version). Computer Science Research Repository arXiv:1307.7066, 2013.

[13] Valmari, A. The asymptotic behaviour of the proportion of hard instances of the halting problem. In Kiss, Á., editor, Proceedings of SPLST '13, 13th Symposium on Programming Languages and Software Tools, pages 170-184, Szeged, Hungary, 2013. 